\title{
IMPROVEMENTS FOR THE WORKFLOW INTEROPERABILITY BETWEEN BIM AND FEM TOOLS
}

\author{
ANDERS SAGVÅG BIRKEMO, SIMEN CHRISTENSEN HJORTLAND \\ \& S. M. SAMINDI M. K. SAMARAKOON \\ Department of Mechanical and Structural Engineering and Materials Science, University of Stavanger, Norway
}

\begin{abstract}
Transferring models, developed using Building Information Modelling (BIM), to Finite Element Method (FEM) tools is an important task in the integration of structural engineering into the open BIM workflow. Such integration saves the time spent by a structural engineer modelling a structure from scratch. There are various problems that can arise while transferring from BIM to FEM tools. A common problem in transferring the geometry and data from the BIM model into an FEM tool is the question of where to place nodes. Each column, beam or slab is defined as a volume in the BIM model and must be interpreted as lines and areas in the FEM analysis. This can be problematic, as transfer of forces must happen at a singular point in the FEM analysis, while the BIM model may be ambiguous regarding where these points are to be placed. This paper discusses the problems that occur when transferring a model from BIM to FEM tools and how to reduce some common mistakes by improving the existing workflow. Based on the needs of local Norwegian industries, the study mainly focuses on the transference of analytical models from Revit software to FEM tools such as Focus Konstruksjon, Robot Structural Analysis and SOFiSTiK. Then, improvements for the existing workflow are proposed for the aforementioned interoperability between BIM and FEM tools and verified using a case study. Keywords: Building Information Modelling, Finite Element Methods, workflow.
\end{abstract}

\section{INTRODUCTION}

The Architecture, Engineering and Construction (AEC) industries have been dependent on paper-based modes of communication; such paper-based documents can cause errors and omissions, resulting in rework, unanticipated cost and delays in construction projects [1]. For example, in Norway, $25-30 \%$ of construction costs are due to fragmentation of processes and poor communication [2]. In the case of implementing Building Information Modelling (BIM), expenses can be paid back in multiples, as it enables a significant reduction in wastage of time and resources. In order for BIM to be effective, it is important to share information with other participants in the AEC industries. For example, it is vital to exchange information of a building analysis and design process, in which many changes can occur based on the requirements of other involved parties. Moreover, during a structural analysis and design process, a structural engineer creates an analytical model (AM) in Finite Element Method (FEM) software, based on information given by the architect (e.g. drawings, initial dimensions and section sizes, etc.) and based on his judgement and experience (e.g. how to create the analytical model, how should the loads be applied, etc.) [3]. However, updating the analytical model manually whenever there are design changes is time-consuming and difficult. In this case, it is vital to have a mechanism to transfer/import models from BIM to FEM software, which reduces the time taken to create a new model from scratch.

Various authors in the literature have discussed interoperability between BIM and FEM tools. For example, Dravai et al. [3] stated that both the Revit-Robot Structural Analysis (RSA) and Tekla Structures Learning-RFEM links showed great potential, as they were able to transfer all the information necessary for structural analysis in an easy and fast way. However, although interoperability of BIM and FEM software exists, a structural engineer 
faces difficulties if the architecture does not follow right workflow. One of the reasons for this is that the design process and workflow needs to change and adapt to a BIM-based one, and there is strong resistance against this change from many engineers, who insist on sticking with the old design methods.

This paper focuses on how to effectively implement BIM in the structural analysis and design process. Moreover, it discusses important considerations that should be taken into account while modelling analytical models in BIM, using a proper workflow to achieve optimum integration in FEM tools. Interoperability of BIM (i.e. Revit) and FEM software (i.e. Focus Konstruksjon, Robot Structural Analysis and SOFiSTiK) is chosen based on the interest of local Norwegian industry.

\section{USE OF BIM AS A TOOL FOR STRUCTURAL ANALYSIS}

The structural engineer is usually provided with a proposed building design that must be analysed and designed to withstand external loads/actions. If this proposal is delivered as a conceptual BIM model, the work begins with converting the conceptual model into a structural BIM model. Columns, beams, walls and doors must be assigned attributes that define their structural properties. This is a process that can involve cooperation with the architect from the beginning of the conceptual design, especially if the structure is likely to require advanced structural design. When the structural BIM model has been assembled, the structure can be analysed in FEA (Finite Element Analysis)/FEM software. The engineer may choose to define the analytical model within the BIM model or to allow the FEA/FEM software to define it outside the BIM model. Whether it is chosen to define the AM within the BIM model or not, there are a few options for transferring the structure to the FEA/FEM tools, as shown in Fig. 1.

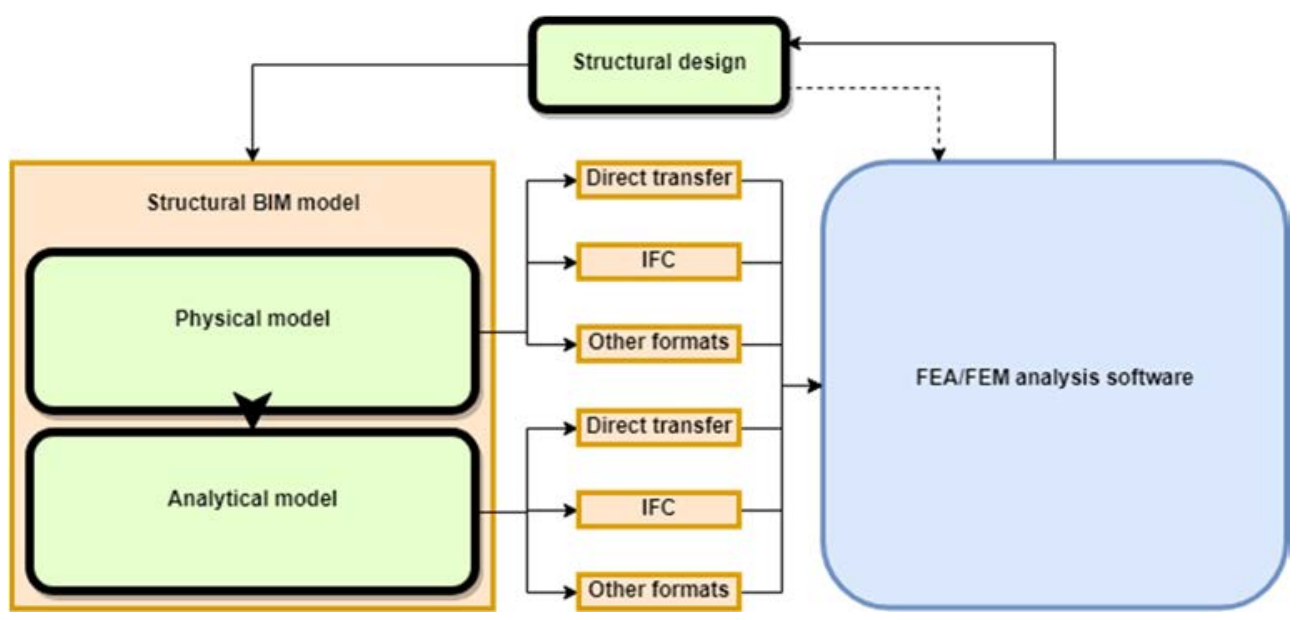

Figure 1: Typical BIM to FEM workflow.

Norwegian AEC industries widely use software, Focus Konstruksjon, SOFiSTiK and Robot Structural Analysis (RSA), in structural analysis and design. Before starting the analysis of structures, it is vital to transfer the AM to the aforementioned software. It is vital to follow the workflow of each type of software. For example, Focus Konstruksjon can accomplish direct transfer of data, as shown in Fig. 2. However, significant differences 
can occur between the AM in Revit and in Focus. Also, the work is somewhat detached from Revit, in the sense that once the data is transferred, the Revit model is "forgotten" until the analysis is done (one-way communication). However, RSA allows for the direct transfer of data. The Revit link option generates a close relationship between the Revit model and the analysis RSA (two-way communication). The workflow of such a level of integration is shown in Fig. 3. SOFiSTiK allows for direct transfer of data. It is even possible to analyse forces and design members from within Revit and; such a level of integration is shown in Fig. 4 (two-way communication).

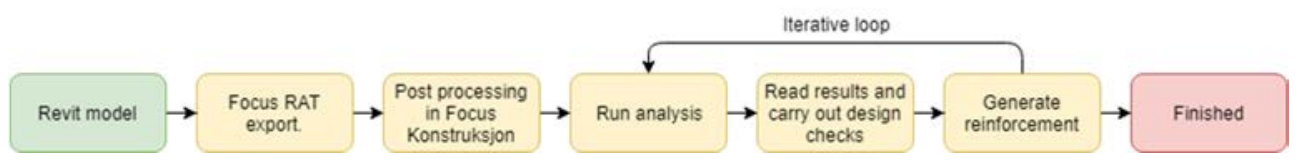

Figure 2: Focus Konstruksjon workflow.

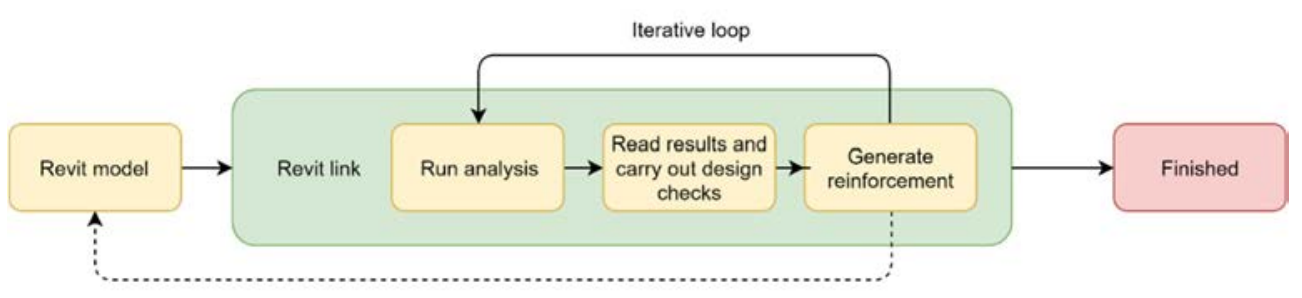

Figure 3: RSA workflow.

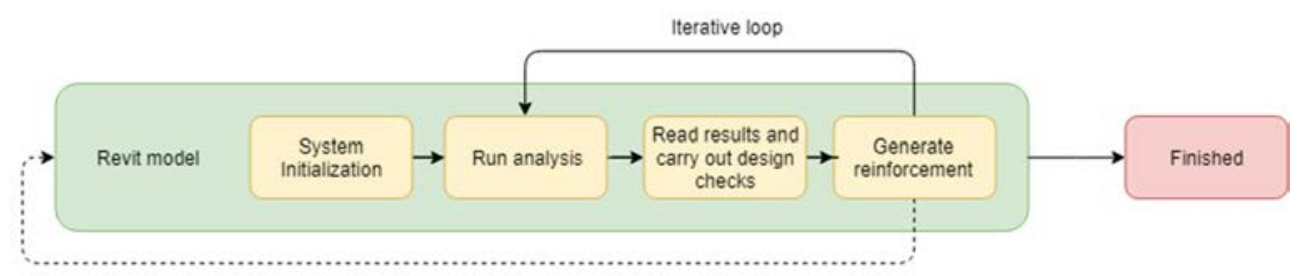

Figure 4: SOFiSTiK workflow.

\subsection{Industrial challenges}

One challenge that structural engineers face while working with BIM models is achieving integrity in the analytical model (AM), which represents the structural system derived from the BIM model. If the modelling work is done without proper attention to the AM, the analysed BIM model might contain analytical discontinuities in the connections between the structural elements. Overcoming these discontinuities can be a time-consuming task, forcing many projects to remake the entire structure within the FEA software.

Using case studies, this study has identified failure modes by observing the behaviour of the AM in response to different modelling practices. One of the case studies is Arkivenes Hus, Stavanger, Norway, as shown in Fig. 5. Arkivenes Hus is a combined and an archive 
building; it has around $10,700 \mathrm{~m}^{2}$ of floor space, approximately $6,200 \mathrm{~m}^{2}$ of which is storage space for the Norwegian National Archives. It was completed in 2017, and the structural design of the building was conducted by one of the consultant companies, Niras Norge AS, Stavanger, Norway, along with some subcontractors. The floors below ground are built up of prefabricated concrete floor slabs, resting on prefabricated concrete beams and concrete columns. The floors above ground are modelled as cast in situ concrete on steel beams and columns. In this case study, Revit software has been transferred to FEM tools, Focus Konstruksjon, Robot Structural Analysis and SOFiSTiK. The initial model in Revit is improved until signs of reasonable behaviour can be shown in the analytical model.

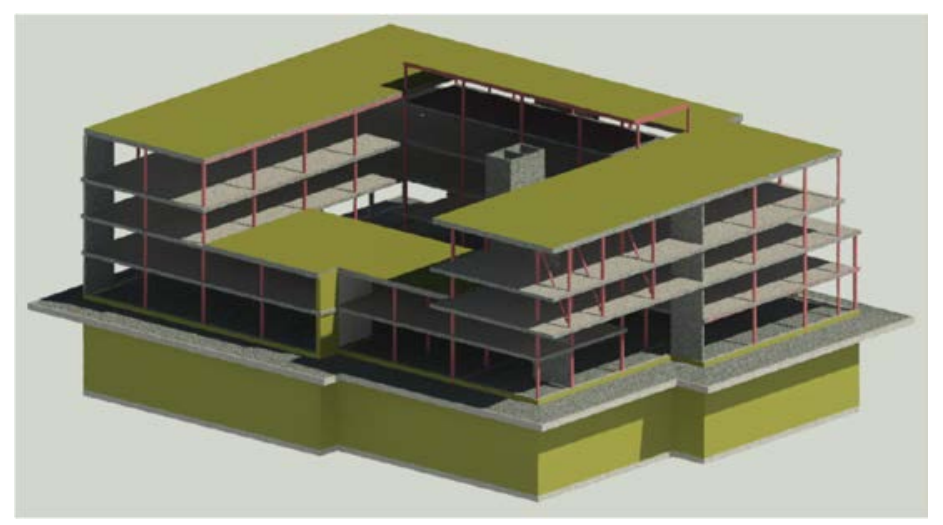

Figure 5: Revit model of Arkivenes Hus building.

Table 1 gives a summary of findings after applying the workflows of different software (i.e. Figs 2, 3 and 4) for the Arkivenes Hus building. According to the results, it can be seen that, if the Revit model does not model considering proper workflow, it results in a lot of errors after transferring to FEM tools. Furthermore, it is quite time-consuming to adjust errors in FEM tools. The success and accuracy of any analysis relies on the AM being consistent and correctly assembled. On inspection, the model of Arkivenes Hus had a variety of problems, such as discontinuity in connection points (e.g. slab to column), overlap of line segments (e.g. prefab slabs overlapping) and continuous columns going through several floors. These are all errors that will either prevent the analysis from starting or severely skew the results to a point where they no longer represent the stresses that would occur in the building.

As previously mentioned, if the BIM model is to be used for structural analysis, it is heavily reliant on the AM being intact and accurately representing the real-world structure. The most common problems were that the nodes that connected the structural elements together were either misaligned or not properly connected. One way of overcoming these problems was to constrain every structural element to the given grid and level lines and, by that, force the structure to achieve the desired integrity. This practice was used to eventually get the Arkivenes Hus model to behave reasonably in SOFiSTiK. However, since these practices had not been followed from the start, there might still be problems which were not identified by the investigation. For that reason, it leads to the conclusion that preventive measures must be taken beforehand, to avoid ending up with a corrupted analytical model. Such measures have been summarized as a set of guidelines, based on our findings; these aim to serve all parties involved in constructing the BIM model. 
Table 1: Summary of challenges and solution.

\begin{tabular}{|c|c|c|}
\hline Workflow & Challenges & Solution \\
\hline Revit to Focus & $\begin{array}{l}\text { - Unable to find steel cross } \\
\text { section profiles in Focus RAT } \\
\text { Experienced instability of the } \\
\text { model } \\
\text { - Unable to export some of the } \\
\text { structural elements due to } \\
\text { inaccuracies in the AM } \\
\text { - } \begin{array}{l}\text { Discontinuity in AM } \\
\text { Missing common node in } \\
\text { shell } \rightarrow \text { line intersection }\end{array} \\
\end{array}$ & $\begin{array}{l}\text { - } \begin{array}{l}\text { Manually define the } \\
\text { cross-sections }\end{array} \\
\text { approximately } \\
\text { - } \quad \begin{array}{l}\text { Not able to fix the } \\
\text { problem due to }\end{array} \\
\text { software limitations } \\
\text { - } \begin{array}{l}\text { Manual placement of } \\
\text { nodes }\end{array} \\
\text { - } \begin{array}{l}\text { Manual remodelling of } \\
\text { problematic areas }\end{array} \\
\end{array}$ \\
\hline $\begin{array}{c}\text { Revit to Structural } \\
\text { Robot }\end{array}$ & $\begin{array}{l}\text { Unable to find some cross- } \\
\text { section profiles } \\
\text { - Instability of the model }\end{array}$ & \\
\hline Revit to SOFiSTiK & $\begin{array}{l}\text { Missing or dislocation of } \\
\text { nodes } \\
\text { - Missing of line intersection } \\
\text { points }\end{array}$ & $\begin{array}{l}\text { - Manual placement of } \\
\text { nodes } \\
\text { - Adjusted using } \\
\text { Analytical Check } \\
\text { feature in the } \\
\text { SOFiSTIK ribbon UI }\end{array}$ \\
\hline
\end{tabular}

\section{SUGGESTION FOR EXISTING PRACTICES OF WORKFLOW}

Based on the challenges, solutions and experience gained from working on the Arkivenes Hus BIM model and other simple models, the workflow suggestions have been drawn up. They are a summary of good practices that have been found to reduce the risk of producing BIM models that contain analytical models with problematic behaviour in a structural analysis context. The case solutions where the analytical model was made compatible with FEM analysis all share the property of having most of these suggestions covered where they are applicable. Some software will also aid the user in achieving the completion of these points and therefore create a greater chance of the successful transfer of information. It is observed that these mainly aim to restrict the analytical representations (segments and shells) of the different elements to certain grid and/or level lines. The accompanying volumetric elements are therefore free to be set for a more accurate representation of the realistic location of the object. This is important when the model is to be used in a collaborative environment and for optimizing the model for collision checks at a later stage.

The main objective of this workflow is to reduce the occurrence of problematic behaviour in the analytical model. Since all connection points have some relation to a specific grid intersection and level line, the model becomes very customizable. In other words, the workflow encourages the use of parametric principles. For instance, if the height of each floor is to be raised by a certain amount, it would only be necessary to adjust the height of all the relevant level lines accordingly. All the elements which have some kind of relation to these level lines will follow and, presumably, without compromising the analytical model.

This workflow aims to cover the most commonly used load bearing elements, and the structural elements which have been considered in this paper are columns, beams, slabs and 
walls. However, these guidelines are also applicable for elements such as diagonal bracings and prefabricated slabs, as placement of these elements will follow many of the same conditions as for beams. The common goal for all elements is to force the connections between the analytical representations (line/shell) together in a desirable way to enable the AM to be exported and analysed without compromises. This is achieved by constraining them to already predefined grid and level lines. By doing this, the AM is not altered, if the volumetric representations have to be offset to correctly mimic the real-world structure (for collision checks), and misalignments are avoided. Another problem to overcome is that common nodes are not always generated at element intersections; one way of solving this is sectioning the structural elements as follows: columns are sectioned at beam and slab intersections, beams are sectioned at column and beam intersections, slabs are sectioned at beam and wall intersections, and walls are sectioned at beam and slab intersections. By following these suggestions, proper connection between all analytical elements is achieved, as nodes are automatically generated at both ends for line segments and at all corners for shell segments. A graphical representation of these guidelines is shown as a flowchart in Fig. 6.
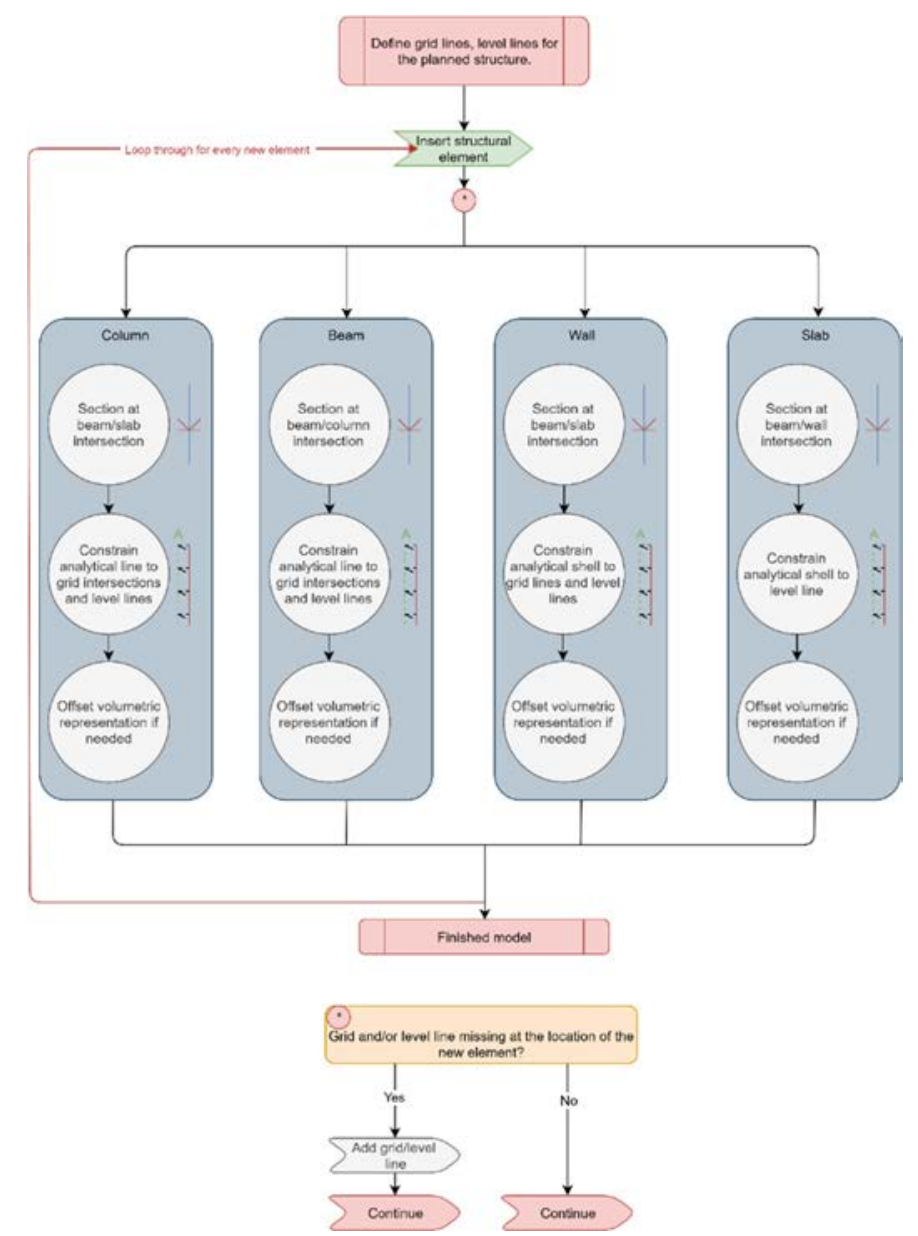

Figure 6: Workflow for generating consistent analytical models. 
A new model with comparable complexity to Arkivenes Hus has been prepared, based on the proposed workflow given in Fig. 6 and the aforementioned guidelines, with the intent of investigating the behaviour of the analytical model while strictly following a proposed workflow. Every single structural element was placed according to the guidelines, and the model was later checked for inconsistencies using SOFiSTiK. According to Fig. 6, for modelling columns, each column was placed one by one in the floor plan view at the relevant grid intersections. Furthermore, each beam was placed one by one in the floor plan view between the relevant grid intersections.

\subsection{Placement of structural elements}

\subsubsection{Columns}

The building consists of columns that span over several floors but intersect beams and slabs, as shown in Fig. 7(b). According to the workflow for modelling columns, each column was placed one by one in the floor plan view at the relevant grid intersections. This was done for all the floors in the building (Fig. 7(a) and (b)). The bases and tops of the columns were constrained to the current level and the level above, respectively (Fig. 7(a)) (base and top constraints). This was repeated for every floor of the building.

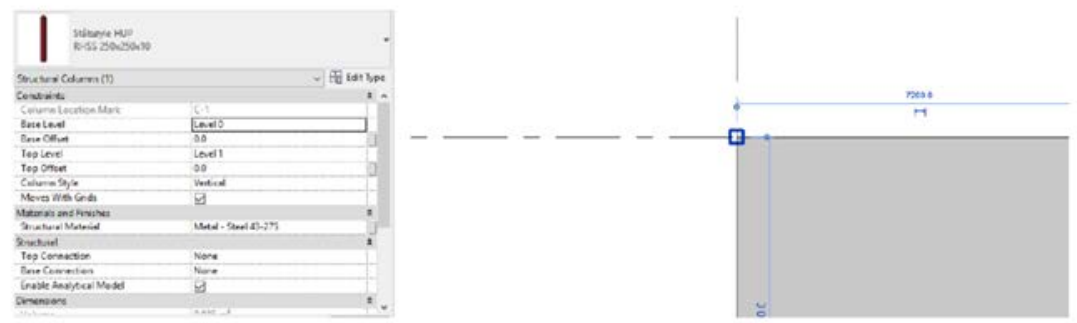

(a)

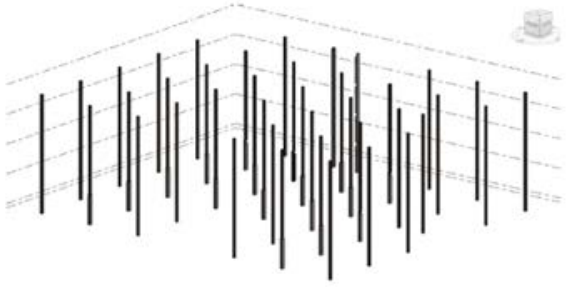

(b)

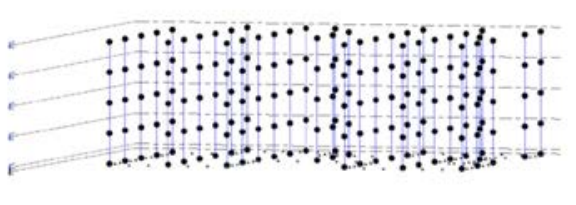

(c)

Figure 7: Placement of a column. (a) Grid intersection in Revit; (b) Volumetric model of columns; (c) Analytical model of columns.

\subsubsection{Beams, prefabricated slabs and diagonal bracings}

The building consists of beams that intersect other beams, columns and slabs. According to the workflow for modelling beams, each beam was placed one by one in the floor plan view between the relevant grid intersections (Fig. 8(a)). The reference level was set to the same as the current floor level (Figs 8(b) and (c)), and this was repeated for every part of the building. 


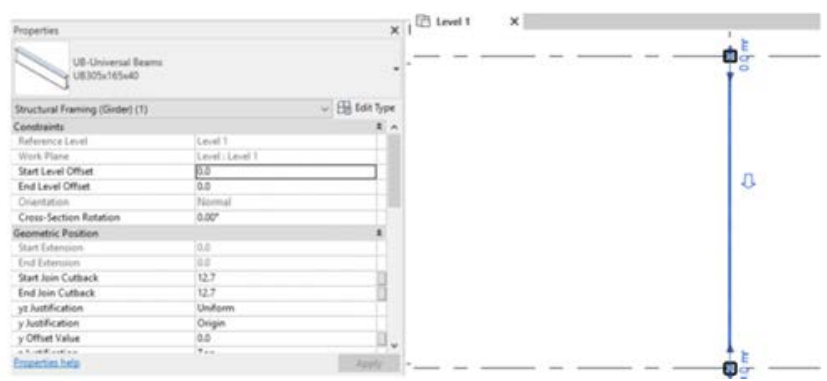

(a)

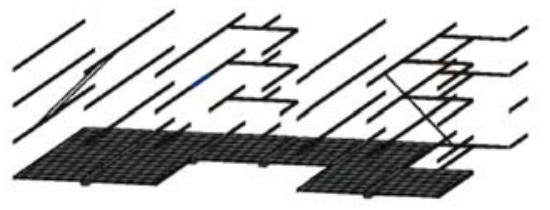

(b)

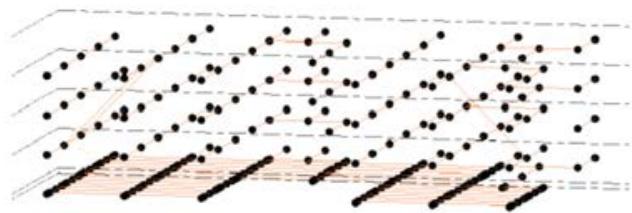

(c)

Figure 8: Placement of beams, prefabricated slabs and diagonal bracings. (a) Placement of a beam, two grid intersections; (b) Volumetric model; (c) Analytical model with sectioned beams.

\subsubsection{Slabs}

The building contains slabs that intersect beams and walls (Fig. 9(b)). According to the workflow for modelling slabs, each slab was placed one by one in the floor plan view between the relevant beam and wall intersections (Figs 9(a) and (b)). The reference level was set to the same as the current one and is applied for every floor of the building.

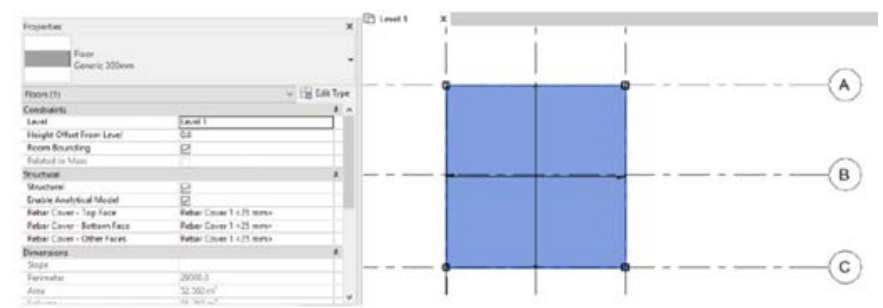

(a)

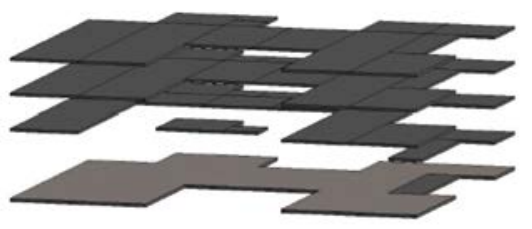

(b)

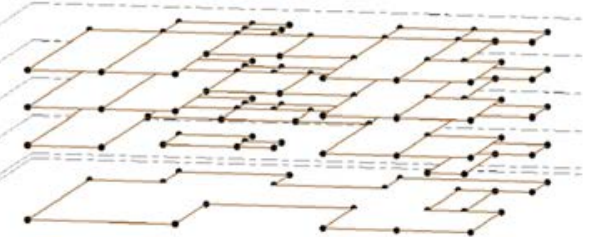

(c)

Figure 9: Slabs in Revit. (a) Placement of slabs; (b) Volumetric model; (c) Analytical model. 


\subsubsection{Walls}

The building contains walls that intersect beams and slabs. According to the workflow for modelling walls, each wall was placed one by one in the floor plan view on the relevant grid line. The bases and tops of the walls were constrained to the current level and the level above, respectively (base and top constraints). This was repeated for every floor of the building.

\subsubsection{Final model}

Fig. 10(a) shows the final model, which is based on the geometry of the physical model and now also contains an AM (Fig. 10(b)) that, according to the workflow, should be consistent; the model was later checked for inconsistencies using SOFiSTiK (Fig. 10(c)).

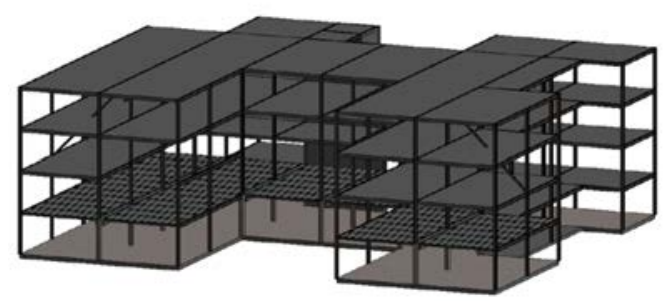

(a)

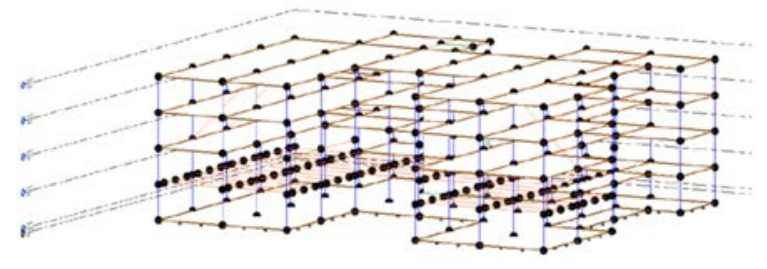

(b)

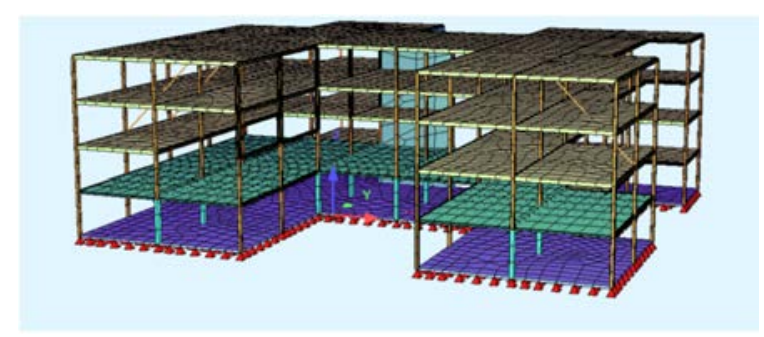

(c)

Figure 10: Final model. (a) Volumetric final model in Revit; (b) The final AM; (c) After analysis of AM in SOFiSTiK.

\section{DISCUSSION AND CONCLUSIONS}

Today, structural engineers may or may not have a generalized set of guidelines for modelling. Implementing a manual/guidelines ensures that all the contributors to the BIM model have reached a certain level of competence. Consequently, misunderstandings are reduced, and it might be easier for different people to pick up on others' work. Finally, the amount of time spent troubleshooting the model will be reduced, as the modelling methods are known to all involved parties. It is vital that all involved parties are familiar with the same 
modelling practices. Many companies already have more or less strict rules for modelling, but these may not be specialized for structural engineering and FEM analysis. If the interoperability between BIM and FEM is increased, time and money will be saved.

The objective of this paper is to suggest a workflow improvement for modelling that facilitates interoperability between BIM and FEM. This has been accomplished in the form of a short and generalized document containing guidelines and modelling rules that could be implemented as a centralized methodology. According to the feedback from NIRAS Norge AS (i.e. Norwegian consultant company), the suggested workflow is a useful set of rules for application in structural modelling. However, some concerns could be raised regarding cross discipline collaboration. These concerns were taken into account in the final version of the workflow. Implementing a manual ensures that all the contributors to the BIM model have reached a certain level of competence. Consequently, misunderstandings are reduced, and it might be easier for different people to pick up on others' work. Finally, the amount of time spent troubleshooting the model will be reduced, as the modelling methods are known to all involved parties.

\section{FUTURE RECOMMENDATIONS}

To further improve and refine the suggested workflow, several areas should be looked in to as given below.

\subsubsection{Use of Dynamo}

Due to the algorithmic nature of the workflow, some or all steps could probably be executed by a Dynamo script. By doing this, the person doing the modelling can rely on the script to somewhat function as a failsafe to ensure mistakes are avoided.

\subsubsection{Use of Autodesk refinery}

Autodesk refinery is a plugin for Dynamo which make use of Machine Learning methodologies to iteratively generate an optimal design according to predefined constraints and free variables. Such technology could be used to enhance and improve the implantation of the guidelines into the BIM-workflow. The software is currently in beta and might be lacking some capabilities at this stage, however, the power of machine learning has proven that it certainly should not be overlooked.

\subsubsection{Need improvements in Autodesk Revit}

To improve the interoperability between BIM and FEA software, Autodesk could improve some aspects of Revit. FEA software that does not offer direct transfer through a Revit plug-in relies on other means of transferring the model. Often IFC is brought up as a valid alternative, however, the analytical model (AM) is not included in the Revit IFC export. This will effectively cause all the work previously set up by the workflow to be void and such FEA software would not draw any benefits from utilizing it. Secondly Autodesk should also improve their toolkit for achieving consistent and corrupt free analytical models, as it is currently not very intuitive and could be more user friendly.

\subsubsection{Further case studies}

To further refine and improve the proposed workflow additional case studies should be modelled and observed for AM integrity. Especially large buildings containing complex geometries are of interest, as these more than likely would shine light on some of the shortcomings of the workflow. Additional findings would be used as inputs for a revised workflow. 


\section{ACKNOWLEDGEMENTS}

The authors would like to thank Helge Mæhle and Stephan Niderehe, NIRAS Norge AS, for providing valuable input and feedback for the proposed workflow.

\section{REFERENCES}

[1] Eastman, C. et al., BIM Handbook, John Wiley and Sons Inc., 2011.

[2] Hjelseth, E., buildingSMART Norge, Presentation on 15 Jan. 2015.

[3] Dravai, T., Khalyar, H. \& Nagy, G., The effect of interoperability between BIM and FEM tools on structural modelling and analysis. Master's thesis, Aalborg University, 2016. 